EPJ Web of Conferences 66, 04013 (2014)

DOI: $10.1051 /$ epjconf/ 20146604013

(C) Owned by the authors, published by EDP Sciences, 2014

\title{
Inhomogeneous chiral phase by a variational approach
}

\author{
S.Karasawa ${ }^{1, a}$ and T.Tatsumi ${ }^{1}$ \\ ${ }^{1}$ Department of Physics, Kyoto University, Kyoto 606-8502, Japan
}

\begin{abstract}
The inhomogeneous chiral phase is discussed in QCD at finite temperature and/or density. We study the phase diagram on the density-temperature plane by taking into account the effect of the current mass by a variational method. It is demonstrated that our framework well describes the inhomogeneous phase over the whole region.
\end{abstract}

\section{Introduction}

Recently the study of the chiral inhomogeneous structure has received much interest in the QCD phase diagram. The chiral inhomogeneous structure is specified by the spatially dependent order parameter of chiral symmetry. Inhomogeneous order parameter has been studied in the condensed matter physics. For example, spin density wave, charge density wave, FFLO superconductor and so on. We study an analogous subject in the quark matter physics. In fact, it has been shown that the chiral inhomogeneous phases exist between the chiral homogeneously broken phases and the chiral restored phases in low temperature and moderate density region of the QCD phase diagram[1-3].

On the other hand, many studies consider only idealized situation; no external magnetic field and no flavor asymmetry, and no current quark mass. External magnetic field and flavor (isospin) asymmetry is important when we consider quark matter in compact stars. Moreover, if we discuss near the critical point, we should consider the effect of the current quark mass carefully.

One way to discuss such effect is a perturbative approach, which consider quark mass term as a perturbation in the thermodynamic potential. This approach is discussed in [4], but we think this approach is not satisfactory way to study the finite mass effect in the "inhomogeneous" order parameter. Because of the finite mass effect, the function type of the inhomogeneous order parameter has changed. Perturbative approach can not trace such transformation.

To trace the change of the thermodynamic potential and the transformation of the function type in the inhomogeneous order parameter, we propose a variational approach. Using a trial function which has already known in the chiral limit, and the stationary condition for the thermodynamic potential, we derive an equation which determine unknown function type of the inhomogeneous order parameter. Since it is hard to solve that equation analytically, we solve it approximately using the derivative expansion. As a result, we derive the sine-Gordon equation and non-linear term in this equation represents the change of the inhomogeneous order parameter from the chiral limit by the effect of the finite current quark mass.

\footnotetext{
ae-mail: karasawa@ruby.scphys.kyoto-u.ac.jp
} 


\section{Framework}

In this section, we introduce a variational approach by using $N_{f}=2, N_{c}=3 \mathrm{NJL}$ model. Here we consider the idealized situation; no external magnetic field and no isospin asymmetry. Lagrangian can be written as ( $m_{c}$ is the current quark mass)

$$
\mathcal{L}_{\mathrm{NJL}}=\bar{\psi}\left(i \not \partial-m_{c}\right) \psi+G\left[(\bar{\psi} \psi)^{2}+\left(\bar{\psi} i \gamma_{5} \tau \psi\right)^{2}\right] .
$$

In previous studies[5], it has been shown that analytic solutions of the inhomogeneous order parameters are no longer real functions but complex functions such as $D(z)=\langle\bar{\psi} \psi\rangle+i\left\langle\bar{\psi} i \gamma_{5} \tau_{3} \psi\right\rangle=$ $\Delta(x) e^{i \theta(x)}$, and represented by the Jacobi elliptic functions in the two dimensional NJL model in the large $N_{c}$ limit. In the chiral limit, we have already known some typical type of condensation. One is called Dual Chiral Density Wave(DCDW), which has spatial dependence only in the phase of $D(x)$. Another one is called Real Kink Crystal(RKC), which has spatial dependence only in the amplitude of $D(x)$. In this study, we assume the function form of the chiral condensate as

$$
\langle\bar{\psi} \psi\rangle=\Delta \cos \theta(z) \quad, \quad\left\langle\bar{\psi} i \gamma_{5} \tau_{3} \psi\right\rangle=\Delta \sin \theta(z) .
$$

Eq. (2) can be understood as a generalization from DCDW in the chiral limit: $\Delta$ is a uniform amplitude of the condensates, and the $\theta(z)$ is a inhomogeneous chiral angle which is now treated as a dynamical variable. Inserting Eq. (2) into Eq. (1) and using the mean-field approximation, the Lagrangian can be rewritten as

$$
\mathcal{L}_{\mathrm{MF}}=\bar{\psi}[i \not \supset-M-U(\theta(z))] \psi-G \Delta^{2},
$$

where $M=m_{c}-2 G \Delta$ is the dynamical quark mass and $U(\theta(z))=-2 G \Delta \exp \left(i \gamma_{5} \tau_{3} \theta(z)\right)-M+m_{c}$. In the Eq. (3), quarks are included in the bilinear form, so that we can integrate them. The mean-field thermodynamic potential then renders

$$
\Omega_{\mathrm{MF}}=-T \operatorname{Tr} \log \left[i \not \partial-M+\mu \gamma_{0}-U(\theta(z))\right]+G \Delta^{2} V,
$$

where the operator trace (Tr) means the sum over flavor, color, Matsubara frequency and integration over the momentum space. $V$ is the volume of the system.

\section{Function form of deformed-DCDW and the thermodynamic potential}

Our main purpose of this paper is to determine the inhomogeneous condensate in DCDW-type and quark wave function with finite current quark mass. Then we introduce the Dirac operator and write the Dirac-Hartree-Fock equations

$$
\begin{aligned}
& H_{\mathrm{D}}=-i \gamma_{0} \gamma \cdot \nabla+\gamma_{0} M+\gamma_{0} U(\theta(z)), \\
& H_{\mathrm{D}} \psi_{\alpha}=E_{\alpha}(\theta) \psi_{\alpha}, \\
& \langle\bar{\psi} \psi\rangle+i\left\langle\bar{\psi} i \gamma_{5} \tau_{3} \psi\right\rangle=\Delta e^{i \theta(z)} .
\end{aligned}
$$

If we can solve Eqs. (6) and (7) simultaneously, we would find the general function form of inhomogeneous condensate and their thermodynamics, but it might be too difficult to solve analytically. Then we find the equation of motion (EOM) for $\theta(z)$ by using the derivative expansion of $\Omega_{\mathrm{MF}}$, and after that, we solve the Dirac equation for quarks using this solution. 
We rewrite $\Omega_{\mathrm{MF}}$ in the form, $\Omega_{\mathrm{MF}}=-T \operatorname{Tr} \log \left[S_{0}^{-1}-U\right]$, Here $S_{0}$ is the free propagator of quarks at finite $T$ and $\mu ; S_{0}(x, y)^{-1}=\left[i \not \partial+\mu \gamma_{0}-M\right] \delta(x-y)$. We invoke the derivative expansion; expanding $\Omega_{\mathrm{MF}}$ with respect to $\partial$ in a Taylor series $[6,7]$

$$
\operatorname{Tr} \log \left[S_{0}^{-1}-U(\theta(z))\right]=\operatorname{Tr} \log \left[S_{0}^{-1}\left(1-S_{0} U(\theta(z))\right)\right] \sim \sum_{n=1}^{\infty} \frac{1}{n}\left(\frac{1}{i \not \partial+\mu \gamma_{0}-M} U(\theta(z))\right)^{n},
$$

where we have neglected $\operatorname{Tr} \log S_{0}^{-1}$ because it has no influence on the EOM of $\theta(z)$. We can regard Eq. (8) as the infinite sum of Feynman diagrams and rewrite them up to $O\left(\partial^{2}\right)$, finally we can approximate the thermodynamic potential

$$
\Omega_{\mathrm{MF}}^{\mathrm{der}}=\frac{1}{2} f_{\pi}^{* 2}\left(\partial_{z} \theta(z)\right)^{2}-m_{c} \Delta \cos \theta(z) .
$$

Here we drop the irrelevant constants for deriving the EOM of $\theta(z)$, and $f_{\pi}^{*}$ is the pion decay constant.

From Eq. (9), we derive the EOM of $\theta(z)$;

$$
\frac{d^{2} \theta(z)}{d z^{2}}-m_{\pi}^{* 2} \sin \theta(z)=0
$$

where we used the Gell-Mann-Oakes-Renner relation, $f_{\pi}^{* 2} m_{\pi}^{* 2}=-m_{c} \Delta$. Eq.(10) is the sine-Gordon (SG) equation. The solution of the $\mathrm{SG}$ equation is given by the $\operatorname{Jacobian}$ amplitude function $\operatorname{am}(x, k)$; $\theta(z)=\pi+2 \operatorname{am}\left(\frac{m_{\pi}^{*} z}{k}, k\right)$ where $0 \leq k \leq 1$ is the modulus parameter.

Once the function form of $\theta(z)$ is obtained, we insert $\theta(z)$ into Eq. (6) to solve these equations, but it is still hard. To circumvent the difficulty, we take notice of the property of $\theta(z)$. From $d \theta(z) / d z=$ $\left(2 m_{\pi}^{*} / k\right) \operatorname{dn}\left(m_{\pi}^{*} z / k, k\right)$, we can see that $\theta(z)$ fluctuates around some mean value. Thus we may expect that the DCDW-type wave function should be suitable for the quark field by replacing the derivative term by its spatial average. Thus we write the quark wave function as $\psi=\mathcal{N} \exp \left(i \gamma_{5} \tau_{3} q z / 2\right) \exp (i \mathbf{p} \cdot \mathbf{r})$, for momentum $\mathbf{p}$ with a variational parameter $q$, which is then related to $k$ by the relation; $q \equiv$ $\left\langle\partial_{z} \theta(z)\right\rangle=\frac{\pi m_{\pi}^{*}}{k \mathbf{K}(k)}$ with the complete elliptic integral of the first kind, $\mathbf{K}(k)$. In this approximation, we find the thermodynamic potential

$$
\begin{aligned}
& \Omega_{\mathrm{MF}} \simeq \Omega_{\mathrm{DCDW}}+\Omega_{\mathrm{SB}}+G \Delta^{2} V, \\
& \Omega_{\mathrm{DCDW}}=-T \operatorname{Tr} \log \left[S_{\text {ref }}^{-1}+\mu \gamma_{0}\right], \\
& \Omega_{\mathrm{SB}}=-T \operatorname{Tr}\left[\left(S_{\text {ref }}^{-1}+\mu \gamma_{0}\right)^{-1} F\left(m_{c} ; \theta(z)\right)\right] .
\end{aligned}
$$

where $S_{\text {ref }}$ is the DCDW-type propagator, $S_{\text {ref }}^{-1}(\theta(z))=i \not \partial-M+q / 2 \tau_{3} \gamma_{5} \gamma_{3}$, and $\Omega_{\mathrm{SB}}$ denotes the symmetry breaking effect of $O\left(m_{c}\right)$. Further detailed discussions would be given in [8].

\section{Numerical results}

In the previous section, we have derived the thermodynamic potential for the inhomogeneous condensate. In this section, we discuss their physical consequences. Minimizing Eq.(11) with respect to $\Delta$ and $k$ with each $T$ and $\mu$, we get the QCD phase diagram in Fig.1.

Similarly to the case in the chiral limit, the inhomogeneous phase appears between the homogeneous condensate phase and chiral restored phase in the QCD phase diagram. The phase transition is of the first order on the left phase boundary.

In the left panel of Fig.2, we see that the modulus $k$ monotonously increases or the wave number $q$ decreases with increasing temperature toward the critical point. The right panel of Fig.2 shows that 


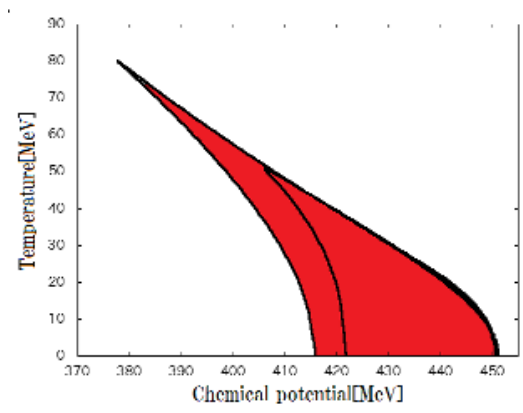

Figure 1. QCD phase diagram at finite temperature and/or density in $m_{c}=0,5 \mathrm{MeV}$. Red region is the chiral inhomogeneous phase. As the current quark mass increases, the area of inhomogeneous phase monotonously decreases..
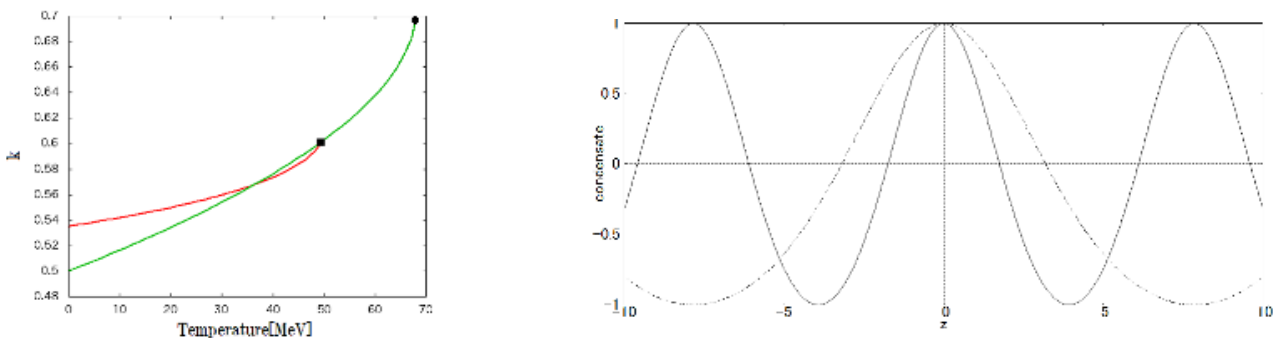

Figure 2. Left: changing of $k$ with increasing $\mathrm{T}$ on the phase boundaries of the inhomogeneous phase. Blue (Red) line shows the left (right) phase boundary. Black dot(square) denotes the CEP("LP"). Right: profile of the scalar condensate $\langle\bar{\psi} \psi\rangle$. Solid line shows the value in the low temperature region, while Dash line the value near CEP. The amplitude of $\langle\bar{\psi} \psi\rangle$ is rescaled.

the scalar condensate oscillates almost periodically in the low temperature region, while it becomes unevenly distributed near the critical point. This uneven distribution can be understood as the consequence of the deformation of the chiral circle; the chiral circle is no more flat in the presence of the mass term: the mass effect makes the absolute minimum on the chiral circle ( $\sim 1$ in Fig.2), so that the condensate favors to stay around this minimum.

\section{References}

[1] D. Nickel, Phys. Rev. D80, 074025 (2009).

[2] E. Nakano and T.Tatsumi, Phys. Rev. D71, 114006 (2004).

[3] G. Basar and G. V. Dunne, Phys. Rev. D78, 065002 (2008).

[4] S. Maedan, Prog. Theor. Phys. 123285 (2009).

[5] G. Basar, G. V. Dunne, M. Thies, Phys. Rev. D79 (2009) 105012.

[6] K.Kikkawa, Prog.Theor.Phys. 56 (1976) 947.

[7] T.Eguchi, H.Sugawara, Phys.Rev. D10 (1974) 4257.

[8] S.Karasawa and T.Tatsumi, arXiv:1307.6448 